ISSN 0103-9954

\title{
PATRÓN ESPACIAL DE LA REGENERACIÓN DE Aspidosperma polyneuron Müll. Arg. EN UN BOSQUE SOMETIDO A APROVECHAMIENTO SELECTIVO EN EL NORTE DE LA PROVINCIA DE MISIONES
}

\section{SPATIAL PATTERN OF REGENERATION OF Aspidosperma polyneuron Müll. Arg. IN A REMNANT SECTOR SUBMITTED TO SELECTIVE USE IN THE NORTH OF MISIONES PROVINCE}

\author{
Martín Pinazo $^{1}$ Fabio Moscovich ${ }^{2}$ Caludio Dummel ${ }^{3} \quad$ Otto Knebel $^{4}$
}

RESUMEN

Se analizó el patrón espacial de la regeneración de Aspidosperma polyneuron Müll.Arg. en un sector remanente de la selva Paranaense en la provincia de Misiones, Argentina. Se mapearon los individuos presentes en un área de $210 \mathrm{~m}$ x $100 \mathrm{~m}$ (2,1 ha). Se aplicaron técnicas de análisis de patrones de puntos univariados y bivariados mediante el índice "O" de Wiegand \& Moloney. La regeneración presentó agrupamientos a escalas de hasta 10 metros resultando en conglomerados de 30 metros de diámetro. No se verificaron asociaciones entre la regeneración y los individuos mayores de la especie ni con los árboles muertos, mientras que se observaron asociaciones a pequeña escala con individuos de otras especies. Los resultados permiten concluir que la regeneración presenta patrones agregados que tienden a la homogeneidad en los adultos. La presencia de renovales está condicionada a la presencia de adultos en las cercanías y la instalación de la regeneración está asociada a individuos de más de $10 \mathrm{~cm}$ de diámetro al pecho siendo la regeneración en sitios abierto una excepción.

Palabras clave: distribución; renovales; Apocynaceae; o estadistico; selva subtropical.

\section{ABSTRACT}

The spatial pattern of regeneration of Aspidosperma polyneuron Müll.Arg. was studied in a remnant sector of the Paranaense forest in the Misiones province, Argentina. All individuals were mapped in a $210 \mathrm{~m}$ x $100 \mathrm{~m}$ area $(2,1 \mathrm{ha})$. Univariate and bivariate point pattern analyses were used by means of Wiegand and Moloney $\mathrm{O}$ ring index. Regeneration showed clumped pattern to scales up to10 $\mathrm{m}$ and clusters of $30 \mathrm{~m}$ of diameter. No association was found between regeneration and both adult trees of $A$. polyneuron and standing death trees, whereas a small scale relationship between regeneration and adult trees of all species was observed. These results revealed that the regeneration of Aspidosperma polyneuron is strongly restricted to the presence of conspecific adult trees. On the other hand, the recruitments are associated with individuals from different species with $\mathrm{DBH}>10 \mathrm{~cm}$ and infrequent in open sites.

Keywords: distribution; saplings; Apocynaceae; o statistic; subtropical forest.

\section{INTRODUCCIÓN}

Uno de los principales objetivos de la ecología de plantas es comprender los mecanismos que producen los patrones de distribución de las mismas en el espacio. Los patrones espaciales reflejan procesos ecológicos implícitos y es por ello que se han desarrollado análisis estadísticos aplicados a la ecología de plantas para poder inferir estos procesos (PERRY et al., 2002). Estos análisis pueden indicar la existencia de procesos que predominan sobre la distribución de las plantas; por ejemplo un patrón regular podría indicar competencia o por el contrario un patrón agregado puede indicar facilitación, sin embargo se debe tener cuidado en la interpretación de los resultado ya que varios procesos pueden generar el mismo patrón espacial (WIEGAND y MOLONEY, 2004). Los patrones espaciales de las plantas pueden generarse a partir de diferentes procesos como la dispersión de las semillas, competición intra e inter especifica, la ocurrencia de eventos extraordinarios o simplemente ser consecuencia de la heterogeneidad ambiental (HUBBEL, 1980; BAROT et al., 1999; CONDIT et al., 2000; WIEGAND et al., 2007). En muchas especies estos procesos

1. Ing. Forestal, M.Sc., INTA-EEA, Montecarlo, Av. Libertador, 2472, C.P. 3384, Montecarlo, Misiones, Argentina. mpinazo@montecarlo.inta.gov.ar

2. Ing. Forestal, Dr., INTA-EEA, Montecarlo, Av. Libertador, 2472, C.P. 3384, Montecarlo, Misiones, Argentina. fmoscovich@montecarlo.inta.gov.ar

3. Estudiante Ing. Forestal, Facultad Ciencias Forestales, Eldorado - UNaM., Argentina. cdummel@yahoo.com.ar

4. Auxiliar, INTA-EEA Montecarlo, Av. Libertador, 2472, C. P. 3384, Montecarlo, Misiones, Argentina.

Recebido para publicação em 7/02/2008 e aceito em 15/05/2009. 
pueden generar patrones regulares o al azar en caso de competencia, como consecuencia de la mortalidad por predación, herbívora o patógenos debido a la manifestación de procesos denso-dependientes (JANZEN, 1970; CONNELL, 1971) o patrones agrupados en el caso de limitaciones en la dispersión de semillas, regeneración en claros o asociaciones entre especies (WIEGAND et al., 2007; SEIDLER y PLOTKIN, 2006; CONDIT et al., 2000); mientras que en otros casos las variaciones en los eventos de fructificación pueden generar patrones al azar o agrupados para una misma especie (HUBBELL, 1980; WRIGHT et al., 2005).

Adicionalmente es posible mediante el ajuste de modelos de patrones espaciales simular los patrones de los procesos de regeneración, ingresos y mortalidad de los individuos de los bosques para su utilización en modelos de dinámica de bosques (BATISTA y MAGUIRE, 1998).

Un método ampliamente aplicado es el estudio de patrones de puntos, que consiste en una base de datos mapeados de una región o área determinada. Existen varios métodos posibles de ser utilizados en la cuantificación de los patrones de puntos (DALE et al., 2002; FORTÍN y DALE, 2005). Los índices estadísticos utilizados pueden ser de primer orden o de intensidad y de segundo orden o de distancia entre pares de puntos. Los estadísticos de primer orden brindan información sobre la intensidad de puntos a gran escala en la región de estudio mientras que los estadísticos de segundo orden, basados en la distancia de pares de puntos, permiten visualizar las relaciones a pequeña escala del patrón de puntos considerado (WIEGAND y MOLONEY, 2004).

Dentro de los métodos desarrollados para analizar la distancias entre puntos se encuentran la función K de Ripley (RIPLEY, 1981) y la función de correlación de pares "G" (DIGGLE, 1983) que utilizan la distancia entre todos los puntos.

El objetivo de los estudios de patrones de puntos es determinar si existe agrupación, regularidad o repulsión o si el patrón es aleatorio. Para ello se construyen limites de confianza generados a partir de los datos observados y comparados contra un modelo nulo simulado mediante simulación Monte Carlo (DIGGLE, 2003). El modelo nulo mas difundido es el CSR (Complete Spatial Randomness) que resulta apropiado en condiciones de homogeneidad en el patrón de puntos (WIEGAND y MOLONEY, 2004). En caso contrario resulta necesario adoptar otro modelo nulo que contemple la condición particular del área en estudio debido a que los efectos a gran escala o de primer orden no permiten definir los efectos de segundo orden o de escala más pequeña, que manifiestan los efectos de la interacción entre plantas (DIGGLE, 2003). Uno de los métodos mas utilizados, si se verifica la falta de homogeneidad, es el Heterogeneous Poisson Process (WIEGAND y MOLONEY, 2004).

El método más conocido para el estudio de patrones de puntos es la función $\mathrm{K}$ de Ripley que presenta la ventaja de ser sensible a desviaciones respecto al modelo nulo (CSR) aunque presenta el problema de adicionalidad ocasionando confusiones respecto a la escala a la cual se producen los efectos de atracción o repulsión entre individuos (WIEGAND y MOLONEY, 2004; CONDIT et al, 2000). Alternativamente la utilización de métodos basados en anillos como por ejemplo la correlación de pares " $g(r)$ " (STOYAN y STOYAN, 1994) o el estadístico O-ring " $O(r)$ " de Wiegand y Moloney (2004) no presentan estos problemas, permitiendo determinar de manera precisa la escala en la cual se manifiestan los procesos (WIEGAND y MOLONEY, 2004; CONDIT et al., 2000).

Aspidosperma polyneuron Müll.Arg. (Palo Rosa) es una de las especies de madera valiosa de la selva paranaense y por lo tanto fue sometida a una fuerte extracción que obligó a la prohibición de su corta. El sistema de extracción selectiva que tradicionalmente se aplica en la provincia de Misiones no contempla aspectos mínimos de manejo del bosque de manera sostenible mas allá de la aplicación de la regla de los diámetros mínimos de corta. Esta práctica no asegura de manera alguna la regeneración y supervivencia de los individuos juveniles de las especies comerciales. Posteriormente a la cosecha forestal es posible verificar una fuerte ocupación del sitio por parte de cañas de las especies Chusquea ramosissima y Merostachys clausenii que junto con la proliferación de lianas Adenocalymna sp. y Arrabidaea sp. impiden la regeneración del bosque por largos periodos de tiempo (CAMPANELLO et al., 2005; CAMPANELLO et al., 2007).

Aspidosperma polyneuron es reconocido como tolerante a la sombra o esciófita por la alta densidad de regeneración en el sotobosque (FONSECA et al., 2004). Diferentes autores clasifican esta especie como secundaria tardía de larga vida (BERNACCI y LEITÃO-FILHO, 1996; MARTINS y RODRIGUES, 2002). 
A pesar de su importancia se dispone de información escasa relacionada a la regeneración de esta especie y a la dinámica espacial de la misma en sitios sometidos a extracción selectiva. Esta información es de suma importancia al momento de generar o aplicar técnicas de manejo que aseguren la sostenibilidad del recurso y la conservación de la especie.

El objetivo del presente trabajo es aportar información respecto a la distribución espacial de la regeneración de esta especie mediante técnicas de análisis espacial de patrones de puntos buscando detectar interacciones entre la regeneración y los individuos mayores de esta especie, individuos de todas las especies y árboles muertos.

\section{Sitio de estudio}

El estudio fue llevado a cabo en el Campo Anexo Manuel Belgrano perteneciente al Instituto

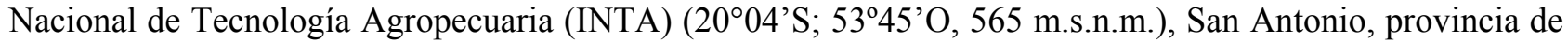
Misiones, Argentina (Figura 1). La temperatura promedio anual es de $20.7^{\circ} \mathrm{C}$, (oscilando entre $25.2-14.8^{\circ} \mathrm{C}$ ). La precipitación promedio anual es de $2108 \mathrm{~mm}$, con un régimen de distribución isohigro.

Como parte de un estudio de dinámica de bosque nativo bajo diferentes manejos se instaló 1 parcela de 2.1 ha $(100 \mathrm{~m} \times 210 \mathrm{~m})$. El sitio fue sometido a extracción selectiva antes de los años 40-50 y posteriormente no se realizaron tratamientos de manejo ni nuevas extracciones. Al momento de instalar dicho ensayo el sitio se encontraba colonizado por una gran cantidad de cañas y lianas.

En cada parcela se realizó la identificación y mapeo de todos los individuos vivos y muertos mayores a 10 cm DAP mediante un cuadriculado de 10 x 10, midiéndose la ubicación de los individuos con cinta métrica. Posteriormente y de la misma manera se relevó y mapeó todos los individuos de Aspidosperma polyneuron cuya altura fuese mayor a 1,3 metros y DAP $<10 \mathrm{~cm}$. (podría ser DAP mayor a $10 \mathrm{~cm}$ (opcional es esto))

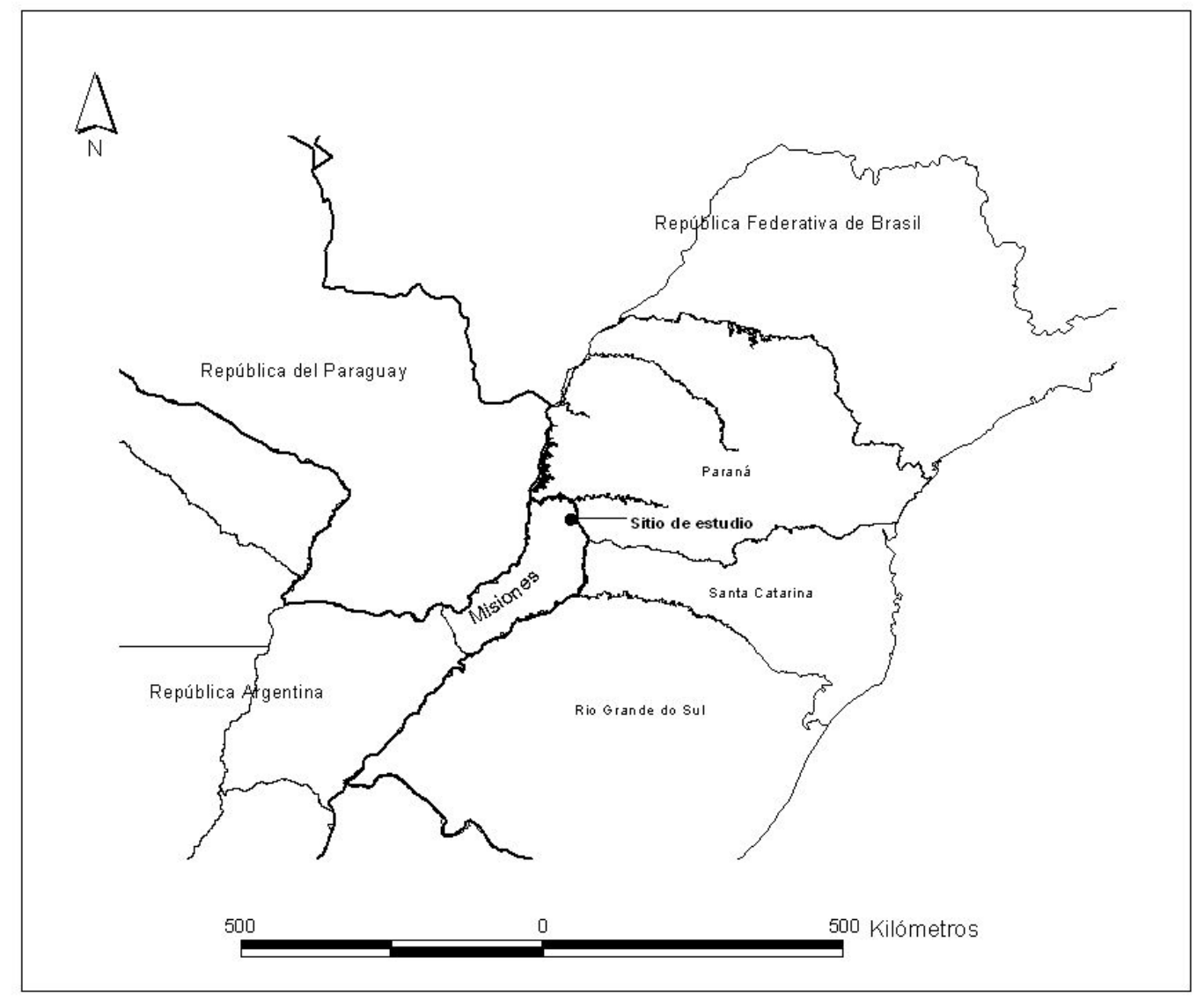

FIGURA 1: Ubicación del sitio de estudio, INTA, San Antonio, Misiones, Argentina.

FIGURE 1: Study site location, INTA, San Antonio, Misiones, Argentina. 


\section{Análisis de datos}

Para el análisis del patrón de los individuos de Aspidosperma polyneuron presentes en la parcela se utilizó el estadístico $O(r)$ desarrollado por Wiegand y Moloney (2004) y el software de análisis de patrones espaciales "Programita" (WIEGAND y MOLONEY, 2004).

El estadístico $O(r)$ es análogo a la función K de Ripley la cual está definida por:

$$
\mathrm{K}(\mathrm{r})=\lambda^{-1} * \mathrm{E}(\mathrm{r})
$$

$\mathrm{E}=$ número esperado de puntos (ej. árboles) a una distancia $\mathrm{r}$ de cualquier otro punto (ej. árboles); $\lambda=$ intensidad de puntos del patrón.

Si el patrón es homogéneo el valor esperado de $K(r)=\pi^{*} r^{2}$

El estatístico $\mathrm{O}(\mathrm{r})$ se estima:

$\mathrm{O}(\mathrm{r})=\mathrm{g}(\mathrm{r}) * \lambda$

Donde $\mathrm{g}(\mathrm{r})$ :

$$
\mathrm{g}(\mathrm{r})=\frac{d K(r)}{d r /(2 \pi r)}
$$

En el caso de patrones bivariados, por ejemplo cuando se analiza la distribución de un patrón (regeneración) respecto a otro patrón (ej: árboles padres), el estadístico se calcula:

$$
\mathrm{O}_{12}(\mathrm{r})=\frac{\frac{1}{n_{1}} \sum_{i=1}^{n 1} \operatorname{Puntos}_{2}\left[R_{1, i}^{w}(r)\right]}{\frac{1}{n_{1}} \sum_{i=1}^{n 1} \operatorname{Area}\left[R_{1, i}^{w}(r)\right]}
$$

Donde: $n_{1}=$ número de puntos del patrón $1 ; R_{1, i}{ }^{w}(r)=$ es el anillo de radio $\mathrm{r}$ y ancho $\mathrm{w}$ centrado en cada punto del patrón 1; Puntos $_{2}=$ Son los puntos del patrón 2 en el área considerada; Área = determina el área de estudio.

El índice $O(r)$ implica la utilización de un anillo de ancho w para cada radio $\mathrm{r}$ y por lo tanto es necesario especificar el ancho de anillo. Esto implica un procedimiento intuitivo ya que no es posible formular reglas para la determinación del mismo. En todo caso el anillo no debe ser demasiado ancho para evitar los problemas de adicionalidad o memoria y tampoco demasiado angosto para evitar perder los efectos en las diferentes escalas (WIEGAND y MOLONEY, 2004). En el presente trabajo se asume un ancho de anillo de 3 metros y el método de corrección de borde propuesto por Wiegand y Moloney (2004).

Se realizaron análisis univariados en el caso de la regeneración y árboles adultos por separado y análisis bivariados explorando las relaciones entre: a) regeneración de Aspidosperma polyneuron-adultos de todas las especies, b) regeneración de Aspidosperma polyneuron-adultos de Aspidosperma polyneuron, c) regeneración de Aspidosperma polyneuron-adultos muertos. Debido a la fuerte agrupación de los renovales observada en la distribución de los mismos en el mapeo, se realizaron los análisis aplicando Heterogeneous Poisson, de esta manera es posible aislar el efecto de la agrupación a gran escala y estimar las distancias a las cuales se manifiestan los procesos. Se utilizó el algoritmo de Epanečnikov para la determinación de la intensidad de puntos recomendado por Stoyan y Stoyan (1994). Como distancia o radio se asumió un valor de 30 ya que en áreas tropicales los procesos de interacción se manifiestan hasta este valor de acuerdo a lo propuesto por Wiegand et al. (2007).

En todos los casos el modelo nulo fue generado respecto al patrón de la regeneración y los limites de confianza se construyeron mediante 999 simulaciones Monte Carlo para obtener una probabilidad del $0.01 \%$ (GOREAUD et al., 1999). De esta manera valores $O(r)>\lambda$ que superen el límite de confianza indican agrupamiento mientras que valores $O(r)<\lambda$ que superen los limites indican repulsión o regularidad. En los casos en los que se verifica una asociación positiva se aplicó el Neyman-Scott Cluster Process para el cálculo del tamaño de los agrupamientos (WIEGAND y MOLONEY, 2004).

\section{RESULTADOS Y DISCUSIÓN}

La distribución espacial de los individuos mayores a $10 \mathrm{~cm}$ de DAP y la regeneración presentados en 
la Figura 2 permite observar la agrupación de los mismos en un sector de área de estudio.

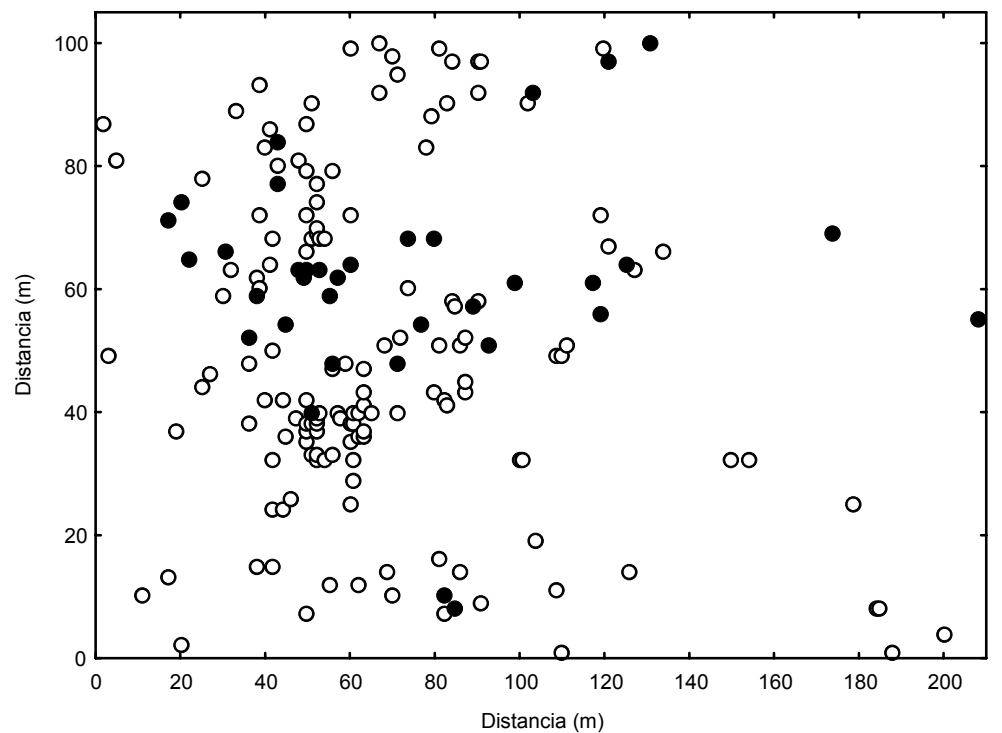

FIGURA 2: Distribución espacial de los individuos de Aspidosperma polyneuron mayores a 10 DAP (círculos negros) y regeneración (círculos abiertos) en el área de estudio.

FIGURE 2: Spatial pattern of individuals of Aspidosperma polyneuron with DBH $>10 \mathrm{~cm}$ (closed circles) and regeneration (open circles) in the area being studied.

Por otra parte, según se observa en la Figura 3, la distribución de frecuencias de los individuos con $\mathrm{DAP} \geq 10 \mathrm{~cm}$ es de forma irregular, no continua, destacándose la existencia de solo 3 individuos con valores de DAP > a $30 \mathrm{~cm}$ y el faltante de ejemplares en las clases diamétricas intermedias. La falta de continuidad podría estar reflejando hasta el presente el efecto del aprovechamiento del área sobre la especie. Como consecuencia del aprovechamiento selectivo concentrado en clases superiores se genero la presencia de pocos individuos de diámetros mayores. Por otra parte y como consecuencia de los claros y daños producto de la intervención es posible que las tasa de mortalidad de individuos maduros o la caída por viento haya aumentado magnificando la disminución de la densidad de individuos en las clases mayores de acuerdo a lo observado en otros bosques tropicales (CANNON et al., 1994). La frecuencia en las clases menores evidencia la buena regeneración de la especie si existen arboles semilleros mientras que la disminución en la frecuencia a medida que aumenta el tamaño de clase manifestaría la intensa competencia inter e intra especifica.

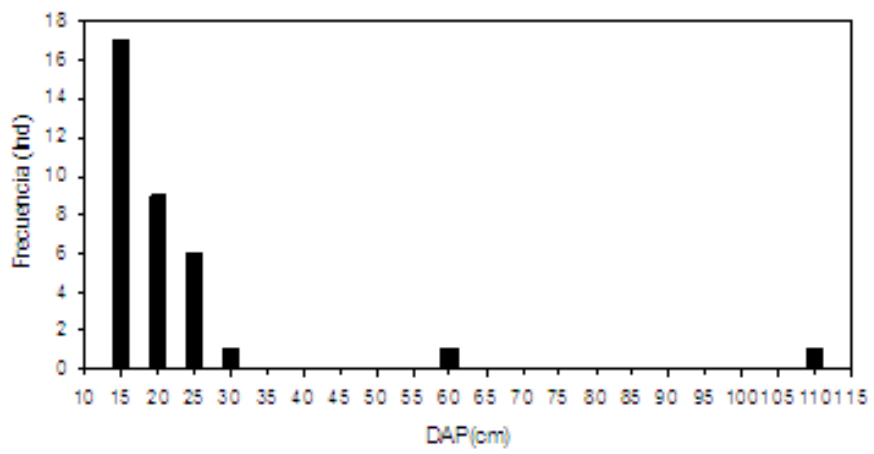

FIGURA 3: Distribución diamétrica de los individuos mayores a $10 \mathrm{~cm}$ DAP de Aspidosperma polyneuron, INTA, San Antonio, Misiones, Argentina.

FIGURE 3: Diametric distribution individuals with DBH $>10 \mathrm{~cm}$ of Aspidosperma polyneuron, , INTA, San Antonio, Misiones, Argentina. 
El análisis univariado del patrón espacial de la regeneración arrojó un agrupamiento significativo a una distancia de $10 \mathrm{~m}$ concentrada a escalas de 5-7 m, mientras que a escalas mayores el patrón resultó al azar (Figura 4). La determinación del tamaño de los agrupamientos de la regeneración resulto en un valor de $30,6 \mathrm{~m}$.

Por su parte, la distribución de los individuos mayores a $10 \mathrm{~cm}$ DAP resultó sin diferencias respecto al modelo nulo en todas las escalas de análisis.
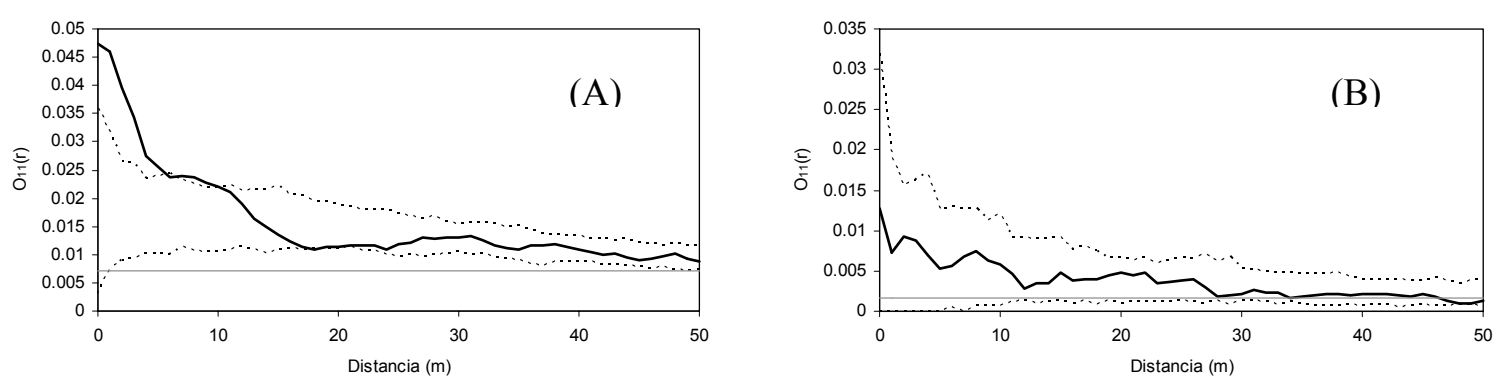

FIGURA 4: Análisis univariado de la regeneración (A) e individuos mayores a $10 \mathrm{~cm}$ DAP (B) de Aspidosperma polyneuron mediante el estadístico " $O(r)$ " de Wiegand y Moloney. En línea punteada limites de confianza al $0.01 \%$; en línea gris " $\lambda$ " o intensidad de puntos del área en estudio; en línea sólida negra valores del $O_{11}(r)$ para las diferentes escalas.

FIGURE 4: Univariate analysis of regeneration of Aspidosperma polyneuron (A), and individuals with $\mathrm{DBH}>10 \mathrm{~cm}(\mathrm{~B})$ by means of the Wiegand and Moloney " $O(r)$ " index. Dashed lines are the $0.01 \%$ confidence limits; grey lines are " $\lambda$ " or the point intensity of the area being studied and the solid lines are the $O_{11}(r)$ values at the different scales.

El patrón general observado de la regeneración de Aspidosperma polyneuron responde posiblemente a efectos relacionados a los aprovechamientos antiguos realizados en el área y a los procesos de dispersión de semillas, las características del dosel circundante y a la presencia de sitios aptos para la regeneración de la especie. Por una parte la distribución diamétrica de los árboles mayores a $10 \mathrm{~cm}$ DAP de Aspidosperma polyneuron muestra claramente el escaso número de individuos de diámetros grandes suficientes para producir grandes cantidades de semillas y al mismo tiempo asegurar la distribución de las mismas en toda el área. Si bien no se dispone de información de edad o tamaño a partir del cual esta especie comienza a producir semilla, es posible asumir que solo los individuos mayores a $30 \mathrm{~cm}$ DAP lo hacen de manera abundante como para condicionar la dispersión en el área. Por otra parte, por presentar esta especie dispersión anemocórica (CARVALHO, 1994), es probable que la regeneración se encuentre asociada en alguna escala a los pocos individuos productores de semillas ya que en general las especies anemocóricas presentan patrones de regeneración agrupados debido principalmente a limitaciones en la dispersión a largas distancias condicionada por la velocidad de los vientos y el dosel circundante (NATHAN y MULLERLANDAU, 2000). Estas limitaciones generan áreas de mayor deposición en cercanía al de los árboles adultos siendo los eventos de dispersión a largas distancias eventos extraordinarios (SEIDLER y PLOTKIN, 2006; CONDIT et al., 2000; NATHAN y MULLER-LANDAU, 2000).

El análisis del patrón espacial de la regeneración mediante el estadístico $O_{11}(r)$ muestra que es posible encontrar agrupamientos de $30 \mathrm{~m}$ de diámetro que significa que esta especie regenera de manera abundante bajo condiciones particulares. Esta característica de agrupamiento de la regeneración también fue observada también por Fonseca et al. (2004) para la misma especie aunque solo pudo determinar agrupamientos de 5-10 m para la regeneración con un diámetro al cuello menor a $1 \mathrm{~cm}$ mientras que no pudo determinar el tamaño de los agrupamientos de las renovales de mayor tamaño. El análisis de los individuos mayores a $10 \mathrm{~cm}$ DAP posiblemente sea la consecuencia de los aprovechamientos realizados en el pasado. Sin embargo la mayoría de los individuos se encuentran por debajo de $10530 \mathrm{~cm}$ DAP y aunque los individuos con DAP $>10 \mathrm{~cm}$ se encuentras restringidos a una porción del área de estudio, condicionando la aplicación del método "Heterogeneous Poisson", los agrupamientos que formaría la regeneración desaparecerían lo cual podría indicar una intensa competencia intraespecifica o la acción de enemigos 
naturales y enfermedades en coincidencia con la teoría denso dependiente o modelo de Janzen-Connell (JANZEN, 1970; CONNELL, 1971). Esta falta de relación entre los individuos mayores también fue reportado por Fonseca et al. (2004).

El análisis bivariado de la distribución espacial de la regeneración de Aspidosperma polyneuron en función de los individuos mayores de la misma especie resultó al azar o no significativo respecto al modelo nulo, indicando la falta de interacción entre ambas categorías. En el caso de la distribución de la regeneración de Aspidosperma polyneuron respecto a los individuos mayores a $10 \mathrm{~cm}$ de DAP de todas las especies el análisis bivariado mostró atracción en escalas pequeñas hasta los $5 \mathrm{~m}$ aproximadamente resultando al azar a escalas mayores (Figura 5).
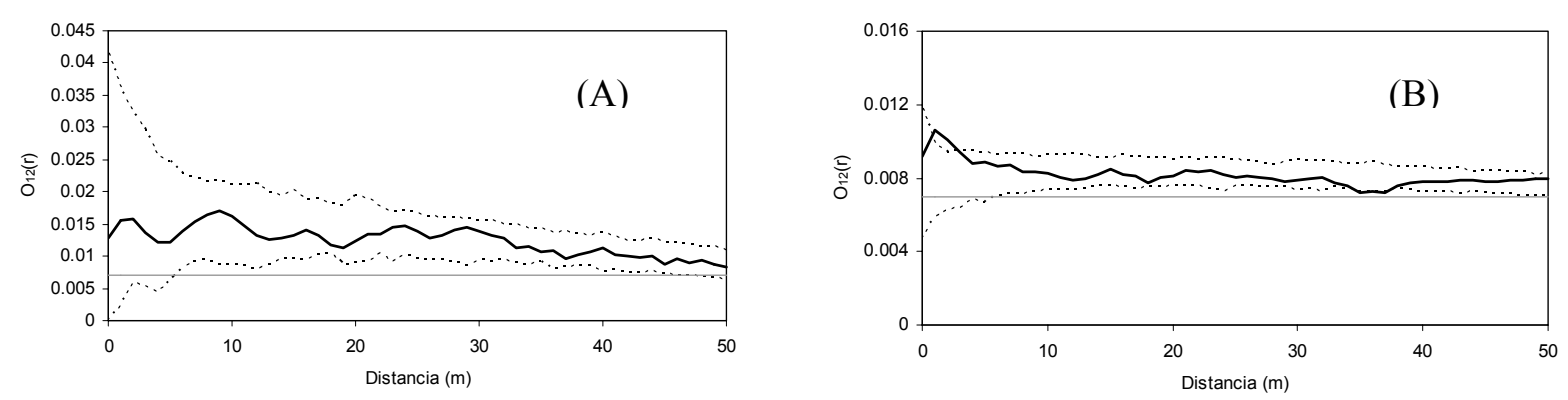

FIGURA 5: Análisis bivariado de la regeneración e individuos mayores a $10 \mathrm{~cm}$ DAP de Aspidosperma polyneuron (A) y de la regeneración de Aspidosperma polyneuron e individuos mayores a 10 cm DAP de todas las especies (B) mediante el estadístico " $O(r)$ " de Wiegand y Moloney. En línea punteada limites de confianza al $0.01 \%$; en línea gris " $\lambda$ ” o intensidad de puntos del área en estudio; en línea sólida negra valores del $O_{12}(r)$ para las diferentes escalas.

FIGURE 5: Bivariate analysis of regeneration and individuals with $\mathrm{DBH}>10 \mathrm{~cm}$ of Aspidosperma polyneuron (A), Regeneration of Aspidosperma polyneuron and individuals with $\mathrm{DBH}>10 \mathrm{~cm}$ of all species (B) by means of the Wiegand and Moloney " $O(r)$ " index. Dashed lines are the $0.01 \%$ confidence limits; grey lines are " $\lambda$ " or the point intensity of the area being studied and the solid lines are the $O_{12}(r)$ values at the different scales.

La ausencia de repulsión o interacción negativa entre ambas categorías en coincidencia con lo reportado por Fonseca et al. (2004) en dos de los sitios estudiados por los autores, refuerza la hipótesis de competencia intraespecifica por sobre la hipótesis de procesos denso dependientes.

La interacción positiva encontrada respecto a los individuos mayores de $10 \mathrm{~cm}$ DAP de todas las especies presentes evidencia mecanismos de facilitación que podrían explicarse por el temperamento de la especie que es clasificada como esciófita. Por otra parte y por tratarse de un sitio sometido a explotación forestal selectiva es posible que el ambiente cercano a individuos mayores tenga menos predominancia de especies invasoras que impidan la regeneración y desarrollo de los renovales y que de esta manera se vean favorecidas las plántulas de esta especie en estos micro ambientes.

El análisis bivariado de la regeneración de Aspidosperma polyneuron y los individuos muertos presentes en el área de estudio no presentaron ninguna relación significativa aunque es posible visualizar una tendencia a la repulsión o interacción negativa entre los 5-15 metros para posteriormente generar una tendencia a la atracción a escalas mayores (Figura 6). La mayor presencia de renuevos de la especie a distancias de $30 \mathrm{~m}$ de los individuos muertos puede explicarse en relación a la característica esciofita de la especie, que prefiere sitios poco expuestos para regenerar (FONSECA et al. 2004). 


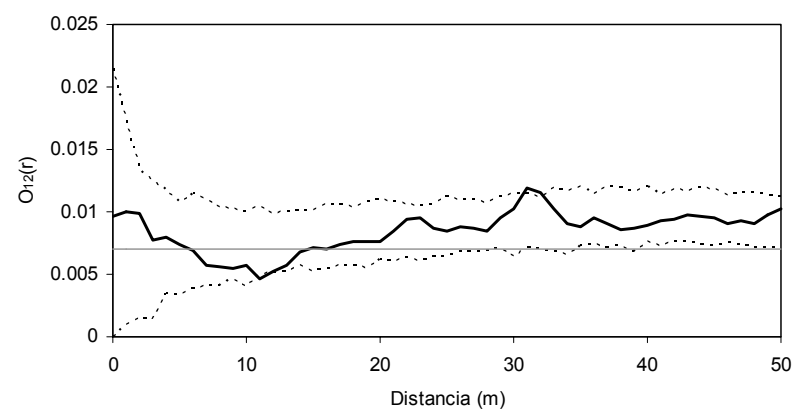

FIGURA 6: Análisis bivariado de la regeneración de Aspidosperma polyneuron e individuos muertos mediante el estadístico " $O(r)$ " de Wiegand y Moloney. En línea punteada limites de confianza al $0.01 \%$; en línea gris " $\lambda$ ” o intensidad de puntos del área en estudio; en línea sólida negra valores del $O_{12}(r)$ para las diferentes escalas.

FIGURE 6: Bivariate analysis of regeneration of Aspidosperma polyneuron and standing death trees by means of the Wiegand and Moloney " $O(r)$ " index. Dashed lines are the $0.01 \%$ confidence limits; grey lines are " $\lambda$ " or the point intensity of the area being studied and the solid lines are the $O_{12}(r)$ values at the different scales.

Si bien la relación con los individuos muertos observada no resultó significativa, la tendencia a la repulsión refuerza la hipótesis sobre las preferencias de ambientes bajo dosel de la especie en su estadio de regeneración.

\section{CONCLUSIONES}

De acuerdo a los resultados observados, es posible aportar información sobre la dinámica de esta especie.

Aspidosperma polyneuron presenta patrones agregados de la regeneración coincidente con otros trabajos. En el caso de los adultos y a pesar de los posibles efectos del aprovechamiento selectivo la tendencia de distribución aleatoria coincide con lo reportado por otros autores. Por otra parte la presencia de renovales estaría fuertemente condicionada por la presencia de adultos debido posiblemente a limitaciones en la distancia de dispersión de las semillas.

A pesar de tratarse de un sitio aprovechado y aunque no se dispone de información sobre el grado de extracción realizado en el pasado, la instalación de los individuos se verifica bajo dosel o en cercanías de árboles mayores a $10 \mathrm{~cm}$ DAP, mientras que la instalación en sitios abiertos tiende a ser una excepción.

En virtud a lo expresado y por tratarse de una especie protegida, es recomendable minimizar el impacto sobre los individuos de esta especie y mantener áreas sin actividades de corta en las cercanías de los árboles maduros de la especie como microhabitats para su regeneración si se pretende recomponer sus niveles poblaciones dentro de un marco de manejo forestal sustentable que contemple la protección de especies en peligro como parte integral de los planes de manejo.

\section{AGRADECIMIENTOS}

A los revisores por su dedicación y valiosos aportes para mejorar el trabajo aquí presentado. El presente trabajo fue financiado en el marco del proyecto INTA MNES06.

\section{BIBLIOGRAFÍA}

BAROT, S.; GIGNOUX, J.; MENAUT, J.C. Demography of a savanna palm tree : predictions from comprehensive spatial pattern analyses. Ecology, v. 80, p. 1987-2005, 1999.

BATISTA, J.L.F.; MAGUIRE, D.A. Modeling spatial structure of tropical forest. Forest Ecology and Management, v.110, p. 293-314, 1998.

BERNACCI, F.C.; LEITÃO-FILHO, H.F. Flora fanerogâmica da floresta da fazenda São Vicente, Campinas, SP. 
Revista Brasileira Botânica, v.19, p.149-164, 1996.

CAMPANELLO, P.; MONTI, L.; GATTI, G.; GOLDSTEIN, G. 2005. Efecto de la tala selectiva sobre la estructura y funcionamiento del bosque nativo en Misiones: Desarrollo de técnicas de manejo forestal sustentable. En: CONGRESO FORESTAL ARGENTINO Y LATINOAMERICANO, 3., 2005, Corrientes. Anales ... Corrientes. Argentina: 2005. $10 \mathrm{p}$.

CAMPANELLO, P. et al. Lianas in a subtropical Atlantic Forest: Host preference a tree growth. Forest Ecology and Management, v. 242, p. 250-259, 2007.

CANNON, C. H. et al. The structure of lowland rainforest after selective logging in West Kalimantan, Indonesia. Forest Ecology and Management, v. 67, p 49-68, 1994.

CARVALHO, P. E. R. Espécies florestais brasileiras: recomendações silviculturais, potencialidades e uso da madeira. Colombo: EMBRAPA - CNPF, Brasília: EMBRAPA - SPI, 1994. 640 p.

CONDIT, R. et al. Spatial pattern in the distribution of tropical tree species. Science, v.288, p. 1414-1418, 2000.

CONNELL, J.H. On the role of natural enemies in preventing competitive exclusion in some marine animals and rain forest trees. En: DEN BOER, P.J.; GRADWELL (ed.) DINAMICS OF POPULATIONS. Wageningn: Prod., 1971. p. 298-312.

DALE, M.R.T. et al. Conceptual and mathematical relationships among methods for spatial analysis. Ecography, v.25, p. 558-577, 2002.

DIGGLE, P.J. Statistical analysis of spatial point patterns. London: Academic Press, 1983. 148 p.

DIGGLE, P.J. Statistical analysis of spatial point patterns. 2. ed. London: Arnold Ed. 2003. 168 p.

FONSECA, M.G.; MARTINI, A.M; DOS SANTOS, F.A. Spatial structure of Aspidosperma polyneuron in two semideciduous forests in Southeast Brazil. Journal of Vegetation Science, v.15, p.41-48, 2004.

FORTIN, M.J.; DALE, M. Spatial Analysis. A guide for Ecologists.Cambride: Cambridge University Press. 2005. $365 \mathrm{p}$.

GOREAUD, F.; COURBAUD, B.; COLLINET, F. Spatial structure analysis applied to modelling of forests dynamics: a few examples. En: Proceedings of the iufro workshop: empirical and process based models for forest tree and stand growth simulation. 1999. p. 155-172.

HUBBELL, S.P. Seed predation and the coexistence of tree species in tropical forests. Oikos, v. 35, p. $214-229$, 1980.

JANZEN, D.H. Herbivores and the number of tree species in tropical forest. The American Naturalist, v.104, p. 501$528,1970$.

MARTINS, S.V.; RODRIGUES, R.R. Gap-phase regeneration in a semideciduos mesophytic forest, southeastern Brazil. Plant Ecology, v.163, p.51-62, 2002.

NATHAN, R., H.C. MULLER-LANDAU. Spatial pattern of seed dispersal, their determinants and consequences for recruitment. Trends in Ecology and Evolution, v.15, p. 277-285, 2000.

PERRY, J.N.; LIEBHOLD, A.M.; ROSENBERG, M.S.; DUNGAN, J.; MIRITI, M.; JACOMULSKA, A.; CITRONPOUSTY, S. Illustrations and guidelines for selecting statistical methods for quantifying spatial pattern in ecological data. Ecography, v.25, p. 578-600, 2002.

RIPLEY, B.D. Spatial Statistics. New York: John Wiley and Sons. 1981. 253 p.

SEIDLER, T.G.; PLOTKIN, J.B. Seed dispersal and spatial pattern in tropical trees. Plos Biology, v. 4, n.11, p. 21322137, 2006.

STOYAN, D.; STOYAN, H. Fractals, random shapes and point fields: methods of geometrical statistics. Wiley: Chichester, 1994. 406 p.

WIEGAND, T.; MOLONEY, K.A. Rings, circles, and null models for point pattern analysis in ecology. Oikos, v.104, p. 209-229, 2004.

WIEGAND, T.; GUNATILLEKE, S.; GUNATILLEKE, N. Species associations in a heterogeneous Sri Lankan Dipterocarp forest. The American Naturalist, v.170, n.4, p.77-95, 2007.

WRIGHT, S.J. et al. Annual and spatial variation in seedfall a seedling recruitment in a neotropical forest. Ecology, v. 86, n.4, p. 848-860, 2005. 